\title{
Egodocuments, biographie et microhistoire en perspective. Une histoire d'amour?
}

Sandro Guzzi-Heeb

\section{(2) OpenEdition}

1 Journals

Édition électronique

URL : http://journals.openedition.org/edl/895

DOI : $10.4000 /$ edl. 895

ISSN : 2296-5084

Éditeur

Université de Lausanne

\section{Édition imprimée}

Date de publication : 15 mai 2016

ISBN : 978-2-940331-48-2

ISSN : 0014-2026

\section{Référence électronique}

Sandro Guzzi-Heeb, «Egodocuments, biographie et microhistoire en perspective. Une histoire d'amour? », Études de lettres [En ligne], 1-2 | 2016, mis en ligne le 01 mai 2019, consulté le 15 décembre 2020. URL : http://journals.openedition.org/edl/895 ; DOI : https://doi.org/10.4000/edl.895

\section{(C) Études de lettres}




\section{EGODOCUMENTS, BIOGRAPHIE ET MICROHISTOIRE EN PERSPECTIVE. UNE HISTOIRE D'AMOUR?}

Depuis les années 1980, la microhistoire a porté une attention renouvelée à la biographie; elle semblait donc prédestinée à se réapproprier les egodocuments. Dans la réalité, le rapport a été plus compliqué et les microhistorien-ne-s ont longtemps privilégié d'autres types de sources. En revenant sur ce rapport complexe, cet article montre que, malgré les difficultés, plusieurs terrains de rencontre entre microhistoire et egodocuments ont été défrichés ces dernières années, à l'instar de l'analyse de réseaux, de l'étude des trajectoires individuelles ou des études d'histoire transnationale. Même en histoire politique, plusieurs historien-ne-s proches de la microhistoire se sont penchés sur des sources du for privé, en proposant de nouvelles voies de lecture "dense». Le rapport entre microhistoire et egodocuments n'a pas dit son dernier mot.

«Partout où les femmes commandent, les choses ne peuvent jamais bien aller». Il y a une dizaine d'années, alors que je travaillais sur la parentèle de la famille valaisanne des de Rivaz, de tels propos, tirés du récit autobiographique de Pierre Emmanuel de Rivaz (1745-1833) ${ }^{1}$ - mercenaire valaisan au service de la France - m'avaient parus significatifs. Ils révélaient à mon sens les contradictions existant entre discours et «réalité» sociale. Le riche fonds de famille de Rivaz, conservé aux Archives d'Etat du Valais, me permettait en effet de comparer ce récit personnel

I. Pierre Emmanuel de Rivaz, Mémoires pour servir à la vie privée et militaire de Pierre Emanuel Jaques de Rivaz ancien Chevalier de l'ordre de Saint Louis et Lieutenant au régiment Suisse de Courten avec rang de Capitaine ensuite Général de Brigade au service de la France écrits par lui-même en 1810, Archives d'Etat du Valais, Fonds de Rivaz, 62. Manuscrit, 1810, p. 90. Je remercie Giovanni Levi pour ses observations critiques et ses suggestions. 
à d'autres témoignages et documents provenant du même milieu, pour situer le jeune de Rivaz dans son contexte familial et social. Or l'analyse montrait que Pierre-Emmanuel avait été en quelque sorte victime de sa famille, et notamment de son influente tante, Marie-Julienne de Nucé. Celle-ci avait pratiquement écarté Pierre-Emmanuel de la succession politique au sein du village, au profit de son propre fils, Charles-Emmanuel de Rivaz. L'émigration militaire avait en fin de compte représenté pour le jeune homme la seule alternative possible. «Partout où les femmes commandent...». A la lumière de son destin personnel, le cliché formulé par Pierre Emmanuel acquiert une toute nouvelle signification.

Dans l'optique d'un historien du social, la contradiction décelée me semblait démontrer à quel point les discours - sur lesquels une grande partie des historien-ne-s se concentraient depuis le succès du "linguistic turn" et des tendances poststructuralistes - pouvaient s'avérer superficiels, voire même trompeurs, face à une réalité familiale et sociale bien plus complexe. Aujourd'hui, une telle interprétation du document me paraît toutefois un peu limitée. Quoique sans velléité de révéler la vérité historique, elle peut nourrir l'illusion selon laquelle seule l'approche par le social - dans mon cas l'approche microhistorique - est en mesure de dévoiler les faits «réels» et les mécanismes d'organisation collective. Sans parvenir toutefois à expliquer, par exemple, pourquoi Pierre-Emmanuel, malgré son destin de victime des logiques familiales, insiste à plusieurs reprises sur les droits des hommes et les hiérarchies familiales «naturelles». L'intérêt de la source autobiographique ne résiderait-il pas, précisément, dans le décalage entre les réalités vécues et transmises par Pierre-Emmanuel et par d'autres membres de son entourage? Quoi qu'il en soit, ces difficultés d'interprétation sont révélatrices d'un rapport plutôt compliqué entre microhistoire et egodocuments: un rapport fait de proximité, d'attirance réciproque, mais aussi de méfiance et de promesses non tenues. Une réflexion sur ce rapport permettra peut-être de mieux saisir les possibilités que ce type de source offre dans une perspective véritablement microhistorique.

\section{La perspective microhistorique des années fondatrices}

Parler aujourd'hui de microhistoire n'est pas chose aisée. Le terme a en effet subi une sorte d'inflation, au point que - dans l'usage courant on ne sait plus très bien ce qu'il veut dire. Preuve en est que des études 
locales menées sur de petites entités, à l'échelle de villages ou de groupes, sont fréquemment assimilées purement et simplement, mais à tort, aux approches « micro».

Il faut dire que la microhistoire n'a jamais été une doctrine, fondée sur une théorie et une pratique universellement partagées. Elle ne repose sur aucun texte fondateur, ne renvoie à aucun dogme partagé ${ }^{2}$. A quelles sources pouvons-nous donc nous référer? Dans les propos qui suivent, j'ai choisi de me baser essentiellement sur les écrits de la première génération des microhistoriens italiens, que je confronterai à des contributions d'autres microhistoriens influents qui ont commencé leur activité à peu près à la même période, dans les années 1980 et au début des années 1990. Cette première génération italienne a notamment pour figures-clés Carlo Ginzburg, Giovanni Levi et Edoardo Grendi. Les deux premiers ont dirigé pendant plusieurs années l'influente collection "Microstorie» chez l'éditeur Einaudi à Turin, qui a notablement contribué à populariser le concept de microhistoire. Edoardi Grendi, peut-être le moins connu des trois - surtout à l'étranger - a apporté une contribution importante à la microhistoire italienne, surtout du point de vue théorique ${ }^{3}$. A côté des éditions Einaudi, qui ont joué un rôle fondamental pour la publication et la diffusion des ouvrages microhistoriques, la revue Quaderni storici, dont Giovanni Levi a été longtemps membre de la direction, a offert aux travaux inspirés par la microhistoire une plate-forme de choix.

Un trait caractéristique de la microhistoire de cette époque a été, évidemment, la réduction de l'échelle d'observation; le groupe social, le village devenaient des laboratoires privilégiés, qui pouvaient révéler des aspects inconnus, voire insoupçonnés de la réalité sociale, en dévoilant de nouvelles dimensions, négligées par les approches plus traditionnelles. Néanmoins, l'horizon des microhistoriens de la première heure n’a jamais été purement local. Il s'agissait plutôt, grâce à la réduction d'échelle, de détecter des éléments historiques invisibles à plus grande distance, mais significatifs sur un plan général. La réduction de l'échelle d'observation se révélait à vrai dire moins centrale que le fait de raisonner

2. J. Revel, «Microstoria», notamment p. 530.

3. V. par ex. E. Grendi, In altri termini et «Ripensare la microstoria?». On pourrait naturellement citer d'autres noms, comme celui de Carlo Poni: C. Ginzburg, C. Poni, "Il nome e il come». 
et de jouer sur des échelles d'analyse différentes ${ }^{4}$. Dans les travaux de l'historien anglais E. P. Thompson, par exemple, traduits et inclus dans la collection "Microstorie", la spécificité de l'approche ne résidait pas dans la taille de l'objet historique - en l'occurrence la culture et l'action des classes populaires -, mais dans la relation de nature anthropologique et critique établie avec l'objet de recherche ${ }^{5}$. Si le fait de travailler sur des entités réduites demeure constitutif des travaux de la plupart des historien-ne-s du courant microhistorique, l'objet de l'étude et sa portée ne se situent pas au niveau local. L'anthropologue Clifford Geertz l'a précisé en des termes qui servirent de programme à un grand nombre de nouvelles recherches: "The locus of study is not the object of study. Anthropologists don't study villages (tribes, towns, neighborhoods ...); they study in villages. " 6

En réalité, malgré un malentendu récurrent, la taille de l'objet observé n'est donc pas essentielle. La différence fondamentale séparant la microhistoire de l'histoire locale traditionnelle se lit ici: dans la capacité de déceler, par une observation "dense» et attentive, des mécanismes historiques qui posent des questions générales, en toute conscience du fait que, dans les différents contextes historiques, ces questions peuvent trouver des réponses divergentes, voire contradictoires. De fait, les microhistoriens visaient depuis le début les grandes interprétations du devenir historique en vogue depuis les années 1960, pour les combattre - pour ainsi dire - en partant d'en bas. Ils étaient mus par un élan critique envers les grands paradigmes de l'histoire politique et sociale de l'époque: contre les théories de la modernisation, les analyses inspirées par l'économie libérale, mais aussi contre les approches d'histoire sociale qui se référaient au fonctionnalisme, au structuralisme et qui avaient trouvé dans l'école des Annales une voix influente et organisée.

L'insatisfaction face aux approches trop généralistes s'était d'ailleurs déjà manifestée à plusieurs occasions; elle se superposait fréquemment à des critiques à l'encontre des positions dogmatiques de la gauche institutionnelle, qui avaient longtemps influencé les sciences sociales. En 1978, le philosophe allemand Lothar Kühne résumait ses doutes en une phrase éloquente: «...une classe sociale en tant que sujet historique ne

4. J. Revel, Jeux d'échelles.

5. E. P. Thompson, Società patrizia, cultura plebea.

6. C. Geertz, The Interpretation of Cultures, p. 22. 
mange pas, ne chante et ne dort pas, et même avec une classe qui lui est amie elle n'aura pas de relations sexuelles... " ${ }^{7}$. Les analyses basées sur des structures rigides de classe et d'action collective manifestaient très clairement leurs limites, non seulement au niveau intellectuel, mais aussi dans la pratique de la lutte politique, face à une société et à une économie de plus en plus complexes.

Plusieurs études avaient d'ailleurs déjà visé les petits espaces, longtemps avant la définition de la microhistoire, afin d'affiner la compréhension d'évolutions historiques significatives ${ }^{8}$. Mais c'est à partir des années 1970 que l'observation attentive des "petites" réalités locales devint un outil servant à déconstruire les implications idéologiques des grandes interprétations de l'histoire.

Face à la crise du structuralisme, beaucoup d'historien-ne-s se tournaient vers d'autres disciplines, à la recherche de nouvelles inspirations. Plusieurs voies méthodologiques s'ouvraient: l'anthropologie historique, l'histoire du quotidien (Alltagsgeschichte), mais aussi l'histoire des femmes et de genre. Toutes remettaient en question les idées et les acquis qui avaient longtemps orienté l'histoire sociale. Plusieurs historien-ne-s privilégiaient ce que Clifford Geerz avait appelé la «description dense» (thick description), soit un regard très attentif au petit détail, à ce qui dérange, et semble au premier abord incompréhensible 9 . Cette approche a explicitement inspiré les microhistoriens allemands de Göttingen, tel Hans Medick ${ }^{10}$ ou Jürgen Schlumbohm. L'exercice pratique de la microhistoire n'en consistait pas moins, pour l'essentiel, et pour tous, en une autre façon de regarder, et une autre façon de décrire ${ }^{11}$. Carlo Ginzburg a essayé de théoriser une approche de ce type en faisant appel à un "paradigme indiciaire» spécifique: le détail incompris, dérangeant, devenant une sorte de signe révélateur (ou «espion»: spia), ouvrant la voie à d'autres significations, d'autres paradigmes et, finalement, d'autres

7. «[...] eine soziale Klasse als gesellschaftliches Subjekt isst, singt und schläft nicht, und selbst zu einer ihr befreundeten Klasse tritt sie nicht in sexuelle Beziehungen [...]»; Cité d'après A. Schnyder-Burghartz, Alltag und Lebensformen, p. 15.

8. Voir par exemple, W. S. Allen, The Nazi Seizure of Power.

9. H. Medick, "Missionare im Ruderboot?" ".

Io. Ibid.

II. Selon l'expression de Giovanni Levi, il s'agissait de «regarder petit»; cité d'après A. Schnyder-Burghartz, Alltag und Lebensformen, p. 13. 
interprétations des sources et de la réalité ${ }^{12}$. Dans cette perspective, l'exception historique devenait donc «normale», puisque la règle présumée était remise en question par l'observation plus attentive. Ce qui apparaissait à première vue comme exceptionnel pouvait être réintégré dans une lecture plus complexe et plus profonde de la réalité. Là résidait au demeurant tout l'intérêt de la recherche historique. Dans la philosophie de la microhistoire - si l'on peut s'exprimer ainsi - l'histoire ne se lit plus selon des schémas linéaires et harmonieux, mais gagne en compréhension par les dissonances, les contradictions, les ruptures, la pluralité des destins et des trajectoires ${ }^{13}$.

L'observation des destins individuels ou particuliers, de leur diversité - ou "variation " ${ }^{14}$ - représentait aussi un élément qui permettait de réinterpréter et de mieux comprendre des activités humaines au-delà de structures rigides qui semblaient les dominer. La diversité des biographies, des perspectives et des trajectoires individuelles et familiales remettait en question la vision de classes sociales soudées par des intérêts communs. Dès lors, le concept de «stratégie» fit figure d'élément central de l'analyse: les individus, loin d'apparaître comme des sortes de marionnettes soumises à de grandes structures qui les dépassaient, retrouvaient une marge de manœuvre personnelle - ou d'agentivité (agency) - qu'ils semblaient avoir perdue dans la vision structuraliste et la "longue durée» braudelienne ${ }^{15}$.

Se basant sur des prémisses similaires, l'historien américain David Sabean a également tenu depuis son premier livre important, Power in the blood (1984), un propos critique à l'égard de la théorie de l'émergence de l'individu occidental émancipé, en s'intéressant à d'autres facteurs de construction et de transformations du «moi» et de l'individualité ${ }^{16}$. Sans liens avec les historiens italiens, étonnamment, Sabean posait une série de questions et soulevait des problèmes très proches des préoccupations de Giovanni Levi qui publiait l'année suivante son livre le plus connu, L'eredità immateriale: que se passe-t-il, dans une société

I2. C. Ginzburg, «Spie».

I3. J. Revel, «Microstoria», p. 532.

I4. F. Barth, "The Problem of Variation».

15. G. Levi, L'eredità immateriale et «I pericoli del geertzismo»; cf. Ch. Delacroix, «Acteur".

I6. D. W. Sabean, Power in the Blood. La critique de la théorie de l'individualisation devient plus explicite dans Kinship in Neckarhausen. 
moderne, quand apparemment il ne se passe rien? Comment s'articule l'Etat au niveau local? Quels sont les effets de l'interaction de pouvoirs différents, et quelles sont les conséquences des frictions avec des cultures populaires? Quelle était la marge pour des stratégies individuelles ou de groupe?

Les stratégies en question, il faut le souligner, n’avaient rien de linéaire. Elles s'adaptaient, d'une part, aux structures existantes, et, d'autre part, aux stratégies des autres acteurs. La microhistoire s'est dès lors montrée très attentive aux interactions à l'intérieur de configurations complexes, dans lesquelles chaque élément influence les autres et interagit avec les autres composantes du système. Cette orientation indiquait déjà l'intérêt de plusieurs microhistorien-ne-s pour l'analyse de réseaux, sur laquelle je reviendrai plus loin. A ce propos, les microhistoriens italiens se référaient à l'anthropologue Frederik Barth et, indirectement, à la théorie des jeux de Morgenstern et von Neumann ${ }^{17}$. Les Italiens demeuraient en effet sceptiques envers le relativisme de Clifford Geertz, qui avait fourni des impulsions très importantes à la microhistoire, surtout en Allemagne: pour Giovanni Levi, tout particulièrement, l'histoire devait impérativement rester une discipline scientifique, en mesure de fournir des indications pour l'interprétation du présent et pour le futur, et donc pour l'action politique ${ }^{18}$. En ce sens, un théoricien comme Barth, très attentif aux problèmes théoriques et aux possibilités de formalisation de l'analyse sociale, était plus proche de certaines positions épistémologiques de la microhistoire italienne ${ }^{19}$.

\section{Microhistoire et egodocuments: des amants prédestinés?}

Les notions de stratégie et d'" agency" poussèrent les historien-ne-s à porter une attention renouvelée à la biographie, longtemps boudée par les intellectuel-le-s des Annales et explicitement proclamée illusoire par Pierre Bourdieu, dans un essai souvent cité ${ }^{20}$. Pour Giovanni Levi, au

I7. F. Barth, «Models of Social Organization I»; O. Morgenstern, J. von Neumann, Theory of Games and Economic Behavior; cf. M. Gribaudi, «Des micro-mécanismes aux configurations globales".

I8. Voir surtout G. Levi, «On Microhistory».

19. F. Barth, Process and Form in Social Life.

20. P. Bourdieu, «L'illusion biographique». 
contraire, la lecture des trajectoires individuelles, avec leurs difficultés, leurs contradictions, leur capacité d'adaptation, enrichissait la compréhension de l'histoire en y réintégrant la dimension subjective ${ }^{21}$. Sabina Loriga - élève de Levi à Turin - a développé et affiné plus récemment cette réflexion sur les interconnexions entre vicissitudes biographiques et "grande» histoire ${ }^{22}$. De ce point de vue, la microhistoire semblait donc destinée à se réapproprier les egodocuments et à en faire une source privilégiée de l'enquête biographique. Dans la réalité, le rapport a été plus compliqué, et l'on peut se demander pourquoi.

Malgré leur intérêt pour l'individu, les microhistorien-ne-s ont longtemps privilégié d'autres types de documents, notamment les sources judiciaires, les actes notariés ou d'autres sources "humbles", jusque-là plutôt négligées, tels que comptes, inventaires, testaments, etc. La microhistoire ne s'est à vrai dire pas concentrée sur un type de source spécifique, elle a plutôt recherché la densité de l'information, l'abondance de sources différentes susceptibles d'affiner et de complexifier l'observation possible d'un objet privilégié. A ce titre, le croisement de sources diverses était privilégié. Les egodocuments n'étaient pas exclus a priori de l'analyse, mais dans la pratique ils ont été considérés plutôt comme des sources historiques parmi d'autres. Par ailleurs, la critique des approches traditionnelles orientait de fait la microhistoire vers d'autres sources, et vers des perspectives qui privilégiaient largement l'étude des couches inférieures, longtemps négligées ou appréhendées collectivement - mais de façon abstraite et impersonnelle - par les approches d'inspiration structuraliste ou fonctionnaliste. Des groupes restés le plus souvent dans l'ombre justement parce qu'ils ne produisaient pas de sources écrites, et restaient tributaires d'une transmission formelle assurée et filtrée par les élites ${ }^{23}$.

Une histoire de populations perdues («lost peoples»), donc? ${ }^{24}$ Au début, depuis I benandanti et Il formaggio e i vermi de Ginzburg, la découverte des cultures, des pratiques et des stratégies populaires a été sans aucun doute un moteur important du renouveau

2I. G. Levi, «Les usages de la biographie».

22. S. Loriga, Le petit $X$.

23. C. Ginzburg, Il formaggio e i vermi.

24. E. Muir, G. Ruggiero, Microhistory and the Lost Peoples of Europe. 
historiographique ${ }^{25}$. Plus tard, toutefois, plusieurs auteurs influents de la microhistoire ont essayé d'ouvrir de nouvelles perspectives par le biais de l'étude de grands artistes ou d'intellectuels, tels que Piero della Francesca, étudié par Ginzburg, ou Galilée, qui a fait l'objet d'un beau livre de Pietro Redondi ${ }^{26}$. En ce sens, la microhistoire se présentait comme une méthode applicable à tout objet historique et à toute classe sociale, se caractérisant par une lecture dense des sources et des contextes y relatifs. L'approche "micro", en d'autres termes, ne se définissait pas par son objet, ni par les sources utilisées, mais par la démarche, par l'attention au détail, au marginal, à l'inconnu.

L'exploitation ciblée d'egodocuments s'y heurtait à plusieurs obstacles. Le premier tenait sans doute à une tradition qui avait traité les sources du for privé plutôt comme des documents littéraires ou d'histoire culturelle ${ }^{27}$. A cela s'en ajoutaient d'autres: d'un côté une (autre) tradition qui associait ce type de source aux grandes théories de la modernisation, notamment au récit de l'émergence de l'individu occidental ${ }^{28}$, et, de l'autre, le problème crucial de la représentativité. Un écueil de taille était en effet lié à la tendance à la généralisation d'exemples spécifiques dans la tradition de l'histoire sociale des Annales. Comme nous l'avons vu, les microhistoriens italiens n'étaient pas les premiers à s'être concentrés sur des villages et de petits groupes pour des recherches historiques significatives. En 1975, Emmanuel Le Roy Ladurie avait par exemple publié Montaillou. Village occitan de 1294 à 1324, qui avait pu être considéré par certains observateurs comme un ouvrage d'orientation microhistorique. En réalité, pour les historiens italiens des années 1970 et 80, Montaillou était le contraire du programme qu'ils prônaient: selon eux, Le Roy Ladurie avait extrait les sources judiciaires concernant les cathares occitans de leur contexte très spécifique, en construisant abusivement sur elles un livre d'anthropologie historique du Moyen Age ${ }^{29}$.

De façon similaire, dans une perspective microhistorique, Daniel Roche tendait à généraliser le récit autobiographique d'un artisan, sans tenir compte de façon adéquate des spécificités individuelles ou

25. C. Ginzburg, Il formaggio e i vermi et I benandanti; cf. plus tard, Storia notturna.

26. C. Ginzburg, Indagini su Piero; P. Redondi, Galileo eretico.

27. J. S. Amelang, The Flight of Icarus, p. 12-16.

28. Voir par exemple R. van Dülmen, Die Entdeckung des Individuums 1500 - 1800 et Die Entdeckung des Ichs.

29. Cf. N. Zemon Davis, «Les Conteurs de Montaillou». 
contextuelles ${ }^{30}$. C'était, à l'époque, une tendance répandue. Il arrivait aussi aux historiens de la famille d'exploiter des sources autobiographiques, telles que les œuvres de Restif de la Bretonne, à titre d'exemple illustrant per se certains phénomènes généraux ${ }^{31}$. La microhistoire avait fortement critiqué cette tendance à considérer des traces fragmentaires comme des illustrations de structures ou grands paradigmes sous-jacents. Prenant le contrepied, elle avait souligné la discontinuité des contextes, la diversité, l'altérité des cultures et des sociétés. Implicitement, elle insistait donc sur l'individualité et la spécificité de chaque source et sur la nécessité d'une critique approfondie de chaque document ${ }^{32}$. Ce qui ne constituait pas une critique fondamentale à l'égard de l'exploitation des egodocuments; un bon usage du point de vue microhistorique des mêmes sources aurait été parfaitement concevable. Ceci dit, des traditions établies poussaient incontestablement les partisans d'une nouvelle histoire, qui se voulait à la fois novatrice et contestatrice, vers d'autres types de sources, à questionner différemment.

\section{Engagement politique et thèmes fédérateurs}

Une caractéristique s'est révélée cruciale dans l'organisation d'une pratique microhistorique: l'engagement politique, soit une proximité évidente des microhistoriens les plus influents avec la gauche, mais dans une posture critique envers les forces institutionnelles du socialisme et du communisme européens. L'effort des microhistoriens italiens de la "première génération" a été clairement de remettre en discussion les interprétations libérales et néolibérales de l'histoire, mais aussi de contribuer à la révision d'une pensée marxiste désormais perçue comme trop rigide et dogmatique. L'ouverture sur l'histoire politique et culturelle, la recherche de la complexité des organisations sociales avaient aussi pour objectif de remettre en question l'influence des lois et des facteurs économiques sur le devenir des sociétés. Que l'un des premiers volumes publiés dans la collection «Microstorie» ait été, comme je l'ai évoqué

30. J.-L. Ménétra, Journal de ma vie.

3I. Voir par exemple J.-L. Flandrin, Les amours paysannes, chap. III; cf. N. E. Restif de la Bretonne, Monsieur Nicolas.

32. Voir plus récemment A. Torre, Luoghi. 
plus haut, la traduction de textes sur la culture et la classe ouvrière de Edward P. Thompson, l'un des marxistes anglais les plus critiques et novateurs ${ }^{33}$, n'est pas un hasard. Aussi, indirectement, l'intérêt politique a constitué un autre facteur d'inspiration commune. Certains historiens ont d'ailleurs essayé de réfléchir aux relations entre histoire, justice et actualité politique, ou sur les utilisations politiques de l'histoire, en proposant des liens avec le présent ${ }^{34}$.

Si la microhistoire n’a jamais été une école organisée, il y a donc eu, en partie au moins, des orientations partagées et des terrains de recherche communs. Depuis lors plusieurs historiens ont par exemple attiré l'attention sur les problèmes de l'évolution de la parenté, qui dans l'esprit des théories de la modernisation et de l'individualisation était censée disparaître, ou s'affaiblir de façon décisive, au cours de l'évolution historique $^{35}$. L'attention à la parenté et à sa persistance apparaissait comme une lecture alternative de l'histoire de la famille et de l'individu, longtemps vus comme les gagnants des transformations historiques qui ont suivi la Révolution française et la révolution industrielle. Ce n'est donc pas un hasard si la question de la parenté à l'ère moderne et contemporaine a représenté un thème en partie fédérateur, d'abord en Italie, plus tard dans d'autres pays ${ }^{36}$. Cet esprit critique, intéressé à la remise en cause d'idées acquises, faisait de ce courant une discipline qui se voulait novatrice et foncièrement expérimentale.

Or, c'est précisément cette volonté iconoclaste de renouveau qui s'est révélée, plus tard, comme l'un des indicateurs d'une crise de la pratique microhistorique. Dans une interview réalisée en 1992, Giovanni Levi déclarait que l'expérience de la microhistoire était achevée, suggérant que le potentiel subversif, novateur de la méthode s'était désormais estompé ${ }^{37}$. La microhistoire a été, de fait, en partie victime de son succès. Une évolution qui dénote quelques limites et contradictions

33. Voir par exemple E. P. Thompson, The Making of the English Working Class et Società patrizia, cultura plebea.

34. C. Ginzburg, Il giudice e lo storico; G. Levi, «Il passato remoto»; F. Hartog, J. Revel, Les usages politiques du passé.

35. G. Levi, «Family and Kin».

36. R. Merzario, Il paese stretto; F. Ramella, Terra e telai; G. Levi, "Tre storie di famiglia"; O. Raggio, Faide e parentela; D. W. Sabean, Kinship in Neckarhausen, 1700 1870; S. Guzzi-Heeb, Donne, uomini, parentela.

37. S. Guzzi, «La storia al microscopio». 
du courant lui-même. La microhistoire n'avait jamais aspiré à se donner une doctrine contraignante. Cette fluidité, sans doute compréhensible, a empêché la définition d'une méthodologie et d'une philosophie commune plus précise. A partir des années 1990, la définition du courant microhistorique a souffert d'une perte progressive de contenu et de profil: pratiquement chaque étude d'histoire locale se voyant attribuer l'étiquette de "micro", quand bien même les approches et les méthodes s'avéraient parfois très traditionnelles. La popularité des approches "micro" contribua ainsi à la fois à en rendre plus nébuleux le profil et à diminuer le contenu novateur des études qui s'en réclamaient ou qui lui étaient rattachées.

A cela vint s'ajouter un second problème, lié à ce qu'on pourrait appeler «le besoin de l'ennemi». Les études microhistoriques ont été d'autant plus significatives qu'elles attaquaient des interprétations ou des théories générales en vogue - théories de la modernisation, évoquées ici à maintes reprises, et leurs corollaires, telle celle de l'émergence d'une famille "moderne" au XVIII e siècle. Après la crise des grandes théories hégémoniques - des Meistererzählungen selon l'expression allemande - la justification d'approches qui s'appliquaient à de très petits groupes ou à des régions limitées est devenue plus problématique. Les auteurs de la première génération ont souvent essayé de formaliser leurs résultats, pour qu'ils deviennent des éléments théoriques significatifs sur un plan plus général: le succès de cette opération a toutefois été partiel et souvent contesté par leurs adversaires ${ }^{38}$. Du reste, même les propositions méthodologiques d'un historien prestigieux comme Carlo Ginzburg, bien que stimulantes, ont plutôt un caractère d'instructions pratiques pour une démarche historique donnée; elles n'ont pas été formulées en tant que théorie cohérente ${ }^{39}$. Ceci dit, si les études microhistoriques restent extrêmement stimulantes, une comparaison de leurs résultats demeure souvent difficile. A ce niveau tout le potentiel de la microhistoire n'a pas encore été épuisé: la réflexion sur le rapport entre échelles d'observations

38. D. Albera, Au fil des générations, p. 32-39.

39. C. Ginzburg, "Spie»; cf. C. Ginzburg, Il giudice e lo storico. Une observation similaire vaut pour d'autres ouvrages d'inspiration microhistorique, par exemple pour A. Torre, Luoghi. Malgré l'intérêt des réflexions méthodologiques de l'auteur, l'interprétation générale du rapport entre production des sources et organisation de l'espace n’apparaît pas pleinement convaincante. 
différentes a été lancée depuis plusieurs décennies ${ }^{40}$, mais elle pourrait à mon sens être sensiblement approfondie.

\section{Microhistoire, egodocuments et analyse de réseaux}

Nonobstant les contradictions et les difficultés indiquées plus haut, plusieurs terrains de rencontre, ou pour le moins de rapprochement possible entre microhistoire et egodocuments ont été partiellement défrichés par les historien-ne-s ces dernières années: je pense notamment à celui de l'analyse de réseaux et de l'étude des trajectoires individuelles. Le concept de réseau est largement entré dans le vocabulaire des sciences sociales depuis plusieurs années. Au départ lié - surtout en sociologie - à la remise en cause de catégories structurelles comme celle de classe, il a plus largement contribué au regain d'intérêt pour les espaces dits «informels», les rapports personnels, ou encore la capacité des individus de construire en partie leur propre milieu. En histoire, l'introduction du vocabulaire des réseaux a souvent été liée à des démarches de prospection à une échelle «micro » et visant à mettre en évidence l'agency individuelle, qu'elles se revendiquent explicitement ou non de la microhistoire italienne.

Ces courants avaient mis un accent particulier sur les notions de configurations de relations, ou par exemple de "fronts de parenté». Si leurs promoteurs avaient connaissance de divers travaux de sociologie portant sur les réseaux, ils n'avaient pour la plupart, dans un premier temps, pas choisi d'utiliser des formalisations graphiques ou des indicateurs chiffrés, considérant qu'il était plus important de cerner de manière qualitative les processus à l'œuvre en insistant sur les facteurs dynamiques ${ }^{41}$. Depuis les années 1990, une analyse de réseaux plus formalisée a fait son apparition dans un autre type de travaux historiques fondés, quant à eux, sur des observations systématiques à une échelle macro: il en va ainsi des analyses de citations en histoire des sciences, de l'étude de parentés et notamment d'alliances sur plusieurs générations en anthropologie historique, ou encore de celle des interlocks, c'est-à-dire des liens entre entreprises

40. J. Revel (éd.), Jeux d'échelles.

4I. M. Bertrand, S. Guzzi-Heeb, C. Lemercier, «Introduction: où en est l'analyse de réseaux en histoire?». Cf. C. Lemercier, «Analyse de réseaux et histoire». 
créés par le partage d'administrateurs, en histoire économique. De telles approches n'ont pas manqué d'exercer une certaine influence sur des études d'inspiration micro-analytique: ces dernières années plusieurs chercheuses et chercheurs ont essayé d'utiliser des méthodes d'analyse formelle pour décrire des phénomènes de petite échelle ou liés à des réseaux égo-centrées, donc autour d'un individu ou d'un groupe particulier ${ }^{42}$. L'étude des trajectoires individuelles a été étroitement liée à celle des réseaux, se focalisant toutefois sur l'évolution des configurations relationnelles et sociales le long des parcours biographiques ${ }^{43}$.

Plusieurs sources du for privé se prêtent à ce type d'exercice: les réseaux de correspondances, notamment, ont été l'objet de plusieurs études qui visaient à rendre visibles des contacts personnels ou individuels, en visualisant des rapports privilégiés.

La lecture des correspondances en termes de réseaux s'est révélée stimulante à des niveaux différents: dans la reconstruction de réseaux savants ou de la transmission de savoirs, par exemple, dans l'interprétation de carrières individuelles, ainsi que dans l'interprétation des relations de personnes alphabétisées dans une certaine mesure "communes". L'étude des réseaux de communication entre intellectuels est devenue entretemps une partie intégrante de l'histoire culturelle. Dans d'autres cas, l'analyse de réseau de correspondances a permis de reconstituer les milieux sociaux de politiciens, hommes d'église, femmes de la noblesse ou de la bourgeoisie ${ }^{44}$.

Pendant les dernières années, les egodocuments ont acquis un rôle tout à fait central dans les études dites d'histoire transnationale ou translocale. L'attention typiquement microhistorique pour les apories de la biographie, pour les parcours individuels a attiré l'attention sur des documents personnels qui témoignaient de trajectoires traversant des espaces différents. Cette orientation, alimentée également par de nouveaux questionnements émergés, depuis les années 1980 dans l'histoire des migrations, a entrainé une remise en question de l'état ou de la nation

42. La référence classique est J. Padgett, Ch. Ansell, «Robust Action and the Rise of the Medici, 1400-1434»; cf. C. Lipp, «Kinship Networks».

43. Voir par exemple C. Bidart, «Dynamiques des réseaux personnels et processus de socialisation".

44. Voir par exemple M. Stuber, S. Hächler, L. Lienhard, Hallers Netz; P.-Y. Beaurepaire, La Plume et la Toile; B. Diaz, J. Siess, L'épistolaire au féminin; cf. J. M. Imízcoz, «Las redes sociales de las élites». 
comme cadres privilégiés de l'étude et de l'interprétation historique. Par une multiplication des études sur les flux migratoires intercontinentaux, sur les réseaux commerçants ou sur les relations internationales depuis le début de l'époque moderne, l'histoire est devenue sensiblement plus translocale, consciente donc de la dimension internationale - ou «interlocale", si l'on veut dépasser la nation comme cadre de référence.

Ce développement a mis au centre de l'intérêt les modalités de contact entre civilisations différentes ainsi que les catégories d'appréhension de l'autre: dans cette perspective, les témoignages directs des acteurs et actrices ont acquis une importance croissante. Plusieurs auteurs ont avancé la nécessité d'une "microhistoire globale», attentive aux témoignages et aux destins individuels, aux contextes locaux et à leurs spécificités, comme instruments privilégiés d'étude et d'interprétation des échanges entre cultures ${ }^{45}$.

\section{Egodocuments et individualisation de la recherche}

Même en histoire politique, après la "période fondatrice", plusieurs historien-ne-s proches de la microhistoire se sont donc rapprochés de l'étude des egodocuments, en proposant des voies de lecture «dense" intéressantes. Récemment, plusieurs études clairement influencées par des approches microhistoriques, ont ouvert de nouvelles pistes ${ }^{46}$. Laurent Brassart a proposé une lecture stimulante des luttes de pouvoir dans un village français à l'époque de la révolution sur la base du récit autobiographique d'un de ses protagonistes. La comparaison d'un récit personnel et d'autres documents concernant le contexte politique de l'époque permet d'éclaircir le poids des motivations personnelles, la dynamique des amitiés et hostilités, des configurations changeantes qui se situent au-delà du domaine proprement politique ou social, dans le sens des intérêts matériels ou de pouvoir ${ }^{47}$. Dans ce cas, la lecture dense

45. F. Trivellato, "Is there a Future for Italian Microhistory in the Age of global History?»; A. Epple, "Globale Mikrogeschichte»; R. Bertrand, L'histoire à parts égales; R. Zaugg, "Johann Peter Oettinger».

46. J. Amelang, The Flight of Icarus, p. 5; voir aussi R. Habermas, Frauen und Männer des Büergertums.

47. L. Brassart, «Plus de vingt paysanneries contrastées en Révolution» et «Devenir républicain pendant l'été $1792 \%$. 
et comparée d'un egodocument permet d'expliquer des phénomènes de nature configurationnelle qui ne seraient pas compréhensibles par une approche sociopolitique classique. Il s'agit à mon sens d'un exemple significatif, permettant de mieux comprendre les mécanismes d'évolution et de diversification de la société.

Les egodocuments - journaux, récits autobiographiques, lettres -, mais aussi certains récits biographiques ${ }^{48}$ peuvent en effet contribuer utilement à la reconstitution de milieux ou de réseaux particuliers ${ }^{49}$. En ce sens ils représentent des sources intéressantes pour de nouvelles démarches qualitatives ou plus formalisées, dans un esprit authentiquement microhistorique.

C'est l'une des directions dans laquelle le travail microhistorique utilisant des egodocuments pourrait évoluer. Plusieurs études publiées ces dernières années ont remis en cause la vision de l'histoire sociale dans la tradition des Annales de grandes transformations globales des sociétés historiques, en suggérant que les grandes évolutions historiques agissent en réalité de façon variée non seulement sur les diverses classes de la société, mais aussi selon des milieux et des groupes culturels différents. La sécularisation, l'augmentation des naissances illégitimes, par exemple, mais aussi l'adoption de la contraception, ne sont pas des phénomènes qui agissent également sur toute la société, ils concernent plus ou moins fortement certaines classes, certains réseaux et certains milieux sociaux et culturels. En ce sens, comme la microhistoire l'avait suggéré, une certaine «individualisation» de la recherche $s^{\prime}$ impose ${ }^{50}$. Il convient de comprendre pourquoi et comment des évolutions touchent certains individus ou groupes plutôt que d'autres. Il ne s'agit plus seulement de remettre l'individu au centre de la recherche, mais plutôt - et cela est essentiel - de comprendre les articulations entre individu, réseaux sociaux particuliers et société, en tenant compte des spécificités des contextes différents. Le concept de milieu me semble crucial dans cette perspective. Il

48. I. Rosé, «Reconstitution, représentation graphique et analyse de réseaux de pouvoir au haut Moyen Âge».

49. Voir par exemple les travaux sur la bourgeoisie allemande: A.-Ch. Trepp, Sanfte Männlichkeit und selbständige Weiblichkeit; D. W. Sabean, "Kinship and Class Dynamics in Nineteenth-Century Europe»; cf. R. Habermas, Frauen und Männer des Büergertums.

50. C. Ginzburg, C. Poni, «Il nome e il come». 
a été souvent utilisé en sociologie pour caractériser des groupes sociaux qui se définissent par de valeurs et des styles de vie similaires. Dans le même sens, j’entends par milieu des réseaux sociaux qui se distinguent par des relations sociales denses, mais aussi par des valeurs, des idées et des attitudes partagées, par exemple sur le plan politique, ou bien, autre exemple, au niveau des comportements sexuels ${ }^{51}$. Un milieu particulier est donc ancré dans un certain groupe social, mais il est en même temps le support d'identités - politiques, religieuses, culturelles... spécifiques même si ce rapport ne doit pas être vu de façon trop rigide.

Le problème qui se pose - et auquel nous n'avons pas encore une réponse convaincante - est celui de savoir comment ces milieux se forment et se reproduisent, comment ils se transforment et se définissent par rapport à d'autres groupes sociaux. Les egodocuments et les correspondances sont, me semble-t-il, des sources précieuses pour comprendre ce type de phénomènes: les annotations personnelles permettent dans plusieurs cas de comprendre comment une personne choisit ses proches - par exemple le futur conjoint - quelles sont les relations privilégiées et quelle sont les frontières invisibles du réseau. Un journal personnel comme celui d'Albertine de Saussure, femme appartenant à la bonne société genevoise de la fin du XVIII e siècle, permet par exemple de mieux appréhender les facteurs qui, dans un certain groupe social, facilitent certaines relations sociales et en entravent d'autres, donc les mécanismes de sélection des proches, de formation du couple et de la famille à l'intérieur d'une société mouvante et complexe ${ }^{52}$. Ce type de document permet probablement de reconstituer des réseaux de sociabilité quotidienne, à l'échelle locale, qui ne laissent pas de traces écrites en dehors des annotations personnelles.

La microhistoire privilégiant la description dense, l'abondance d'informations, le croisement des sources et des perspectives, l'egodocument représente toutefois un point de départ, qui idéalement devrait être comparé à d'autres sources. Cela me semble crucial, afin de pouvoir constituer

5I. Voir par exemple R. Geißler, Die Sozialstruktur Deutschlands, p. 126 sqq.; S. Hradil, Soziale Ungleichheit in Deutschland, p. 425 sqq.; D. W. Sabean, S. Teuscher, "Introduction"; cf. D. W. Sabean, "Kinship and Class Dynamics in NineteenthCentury Europe».

52. A. de Saussure, "Chronique de sa vie quotidienne». Je remercie Sylvie Moret Petrini pour m’avoir indiqué cette source. 
des réseaux suffisamment denses et significatifs, mais aussi afin de comprendre les caractéristiques spécifiques du contexte historique.

Dans cette perspective un récit autobiographique comme celui de Pierre-Emmanuel de Rivaz, mentionné au début de cette contribution, peut devenir le point de départ de maintes interrogations passionnantes, tout à fait dans un esprit microhistorique. Le comparer avec la correspondance familiale, mais aussi avec les autres sources relatives à son contexte social et familial, permet par exemple de reconstituer ce que je qualifierais d'ordre privé de la famille, c'est-à-dire l'ordre et les autorités constituées sur la base des rapports personnels et émotionnels, qui sont dans ce cas nettement différents de l'ordre hiérarchique défini par le droit ou par la coutume. Dans le cas des de Rivaz, la tante de Pierre-Emmanuel - Marie Julienne - a très clairement un rôle crucial et une autorité personnelle indiscutable, alors que les hommes de la famille apparaissent, dans les egodocuments qui nous ont été transmis, dans une position plutôt marginale. Une histoire des émotions, mais aussi une histoire de genre, peut en tout cas difficilement se passer de documents qualitatifs de ce type.

Le récit permettrait même d'aller au-delà de ce constat, pour essayer de reconstituer la formation de l'identité personnelle et sociale de son auteur. Il s'agit par exemple de comprendre, dans une optique translocale, le rapport entre les expériences du soldat et futur général à l'intérieur et à l'extérieur de sa patrie valaisanne; de comprendre comment ses expériences militaires et ses expériences de soldat mercenaire en France se rapportent à son éducation et à sa socialisation primaire et à son réseau valaisan.

La condition sine qua non, quoi qu'il en soit, consiste à comparer les egodocuments avec d'autres types de source, pour parvenir à une lecture et à une analyse dense des trajectoires individuelles, des réseaux sociaux et des identités relatives. Bref, plusieurs pistes de recherche s'ouvrent, qui pourraient nous éclairer sur les logiques qualitatives des réseaux sociaux, sur la constitution des milieux, des identités, sur les émotions, sur les autorités familiales, ainsi que sur les subtils rapports de pouvoir et d'influence dans la sphère privée. Dans cette perspective, l'egodocument pourra peut-être nous livrer des éléments pour une nouvelle "psychologie 
historique», basée non plus sur de grandes généralisations ${ }^{53}$, mais plutôt sur l'analyse dense d'individus et de petits groupes concrets, dans leur contexte spécifique. Bien que parfois difficile, le rapport entre microhistoire et egodocuments n'a donc pas dit son dernier mot.

\section{Sandro Guzzi-Heeb}

Université de Lausanne

53. R. Mandrou, Introduction à la France moderne, 1500-1640. Cf. L. Febvre, «La sensibilité et l'histoire». 


\section{BIBLIOGRAPHIE}

Albera, Dionigi, Au fil des générations. Terre, pouvoir et parenté dans l'Europe alpine, (XVI ${ }^{e}-X X^{e}$ siècles), Grenoble, Presses universitaires de Grenoble, 2011, p. 32-39.

Allen, William S., The Nazi Seizure of Power. The Experience of a Single German Town, 1922-1945, Chicago, Quadrangle Books, 1965.

Amelang, James S., The Flight of Icarus: Artisan Autobiography in Early Modern Europe, Stanford, Stanford University Press, 1998.

Barth, Fredrik, "The Problem of Variation", in Balinese Worlds, Chicago/London, University of Chicago Press, 1993, p. 92-105.

Barth, Fredrik, "Models of Social Organization, I", in Process and Form in Social Life. Selected Essays of Frederik Barth, ed. by Adam Kuper, London, Routledge \& Kegan Paul, 1981, vol. 1, p. 32-47.

Beaurepaire, Pierre-Yves (éd.), La plume et la toile. Pouvoirs et réseaux de correspondance dans l'Europe des Lumières, Arras, Artois Presses Université, 2002.

Bertrand, Michel, Guzzi-Heeb, Sandro, Lemercier, Claire, "Introduction: où en est l'analyse de réseaux en histoire?", in Analyse des réseaux et histoire: outils, approches, problèmes / Análisis de redes e historia: herramientas, aproximaciones, problemas. Redes. Revista hispana para el análisis de redes sociales, éd. par Michel Bertrand, Claire Lemercier, Sandro Guzzi-Heeb, 21 (2011), p. 12-22.

Bertrand, Romain, L'histoire à parts égales. Récits d'une rencontre Orient-Occident, XVIe-XVII siècle, Paris, Seuil, 2011.

Bidart, Claire, «Dynamiques des réseaux personnels et processus de socialisation: évolutions et influences des entourages lors des transitions vers la vie adulte», Revue française de sociologie, 49 (2008), p. 559-583.

Bourdieu, Pierre, "L'illusion biographique», Actes de la recherche en sciences sociales, 62 (1986), p. 69-72.

Brassart, Laurent, "Plus de vingt paysanneries contrastées en Révolution. De la pluralité des dynamiques sociales du politique 
en milieu rural pendant la Révolution ", Annales Historiques de la Révolution française, 359 (2010), p. 53-75.

Brassart, Laurent, «Devenir républicain pendant l'été 1792. Quelques itinéraires individuels dans la France septentrionale», in 1792. Entrer en République, dir. par Michel Biard, Philippe Bourdin, Hervé Leuwers, Pierre Serna, Paris, Armand Colin, 2013, p. 167-184.

Delacroix, Christian, "Acteur", in Historiographies I. Concepts et débats, éd. par Christian Delacroix, François Dosse, Patrick Garcia, Nicolas Offenstadt, Paris, Gallimard, 2010, p. 651-663, (Folio histoire).

Diaz, Brigitte, Siess, Jürgen (dir.), L'épistolaire au féminin. Correspondances de femmes, XVIII ${ }^{e}-X X^{e}$ siècle: actes du Colloque de Cerisy-la-Salle, 1-5 octobre 2003, Caen, Presses universitaires de Caen, 2006.

Dülmen, Richard van, Die Entdeckung des Individuums 1500 - 1800, Frankfurt am Main, Fischer Taschenbuch Verlag, 1997.

—, Die Entdeckung des Ichs: die Geschichte der Individualisierung vom Mittelalter bis zur Gegenwart, Köln, Böhlau, 2001.

Epple, Angelika, "Globale Mikrogeschichte. Auf dem Weg zu einer Geschichte der Relationen", in Im Kleinen das Grosse suche Mikrogeschichte in Theorie und Praxis, hrsg. von Ewald Hiebl et al., Innsbruck, Studien Verlag, 2012, p. 37-47.

Febvre, Lucien, «La sensibilité et l'histoire. Comment reconstituer la vie affective d'autrefois?", in Combats pour l'histoire, Paris, Albin Michel, 1992 (1953), p. 221-238.

Flandrin, Jean-Louis, Les amours paysannes. Amour et sexualité dans les campagnes de l'ancienne France (XVI ${ }^{e}-X I X^{e}$ siècle), Paris, Gallimard/Juillard, 1975.

Geertz, Clifford, The Interpretation of Cultures, New York, Basic Books, 1973.

Geissler, Rainer, Die Sozialstruktur Deutschlands. Die gesellschaftliche Entwicklung vor und nach der Vereinigung, Wiesbaden, Westdeutscher Verlag, 2002 (3. Auflage).

Ginzburg, Carlo, I benandanti. Stregoneria e culti agrari tra Cinquecento e Seicento, Torino, Einaudi, 1966.

-, Il formaggio e i vermi. Il cosmo di un mugnaio del '500, Torino, Einaudi, 1976. 
—, Indagini su Piero, Il Battesimo, il ciclo di Arezzo, la Flagellazione di Urbino, Torino, Einaudi, 1981.

—, "Spie. Radici di un paradigma indiziario", in Miti, emblemi, spie. Morfologia e storia, Torino, Einaudi, 1986, p. 158-209.

—, Storia notturna. Una decifrazione del sabba, Torino, Einaudi, 1989.

—, Il giudice e lo storico. Considerazioni in margine al processo Sofri, Torino, Einaudi, 1991.

Ginzburg, Carlo, Poni, Carlo, «Il nome e il come: scambio ineguale e mercato storiografico", Quaderni storici, 40 (1979), p. 181-190.

Grendi, Edoardo, In altri termini. Etnografia e storia di una società di Antico Regime, a cura di Angelo Torre, Osvaldo Raggio, Milano, Feltrinelli, 2004.

Grendi, Edoardo, «Ripensare la microstoria?», Quaderni storici, 86 (1994), p. 530-549.

Gribaudi, Maurizio, "Des micro-mécanismes aux configurations globales: causalité et temporalité historiques dans les formes d'évolution de l'administration française au XIX ${ }^{\mathrm{e}}$ siècle", in Mikrogeschichte, Makrogeschichte, komplementär oder inkommensurabel?, hrsg. von Jürgen Schlumbohm, Göttingen, WallsteinVerlag, 1998, p. 83-128.

Guzzi, Sandro, "La storia al microscopio", Cooperazione, 23.01.1993.

Guzzi-Heeb, Sandro, Donne, uomini, parentela. Casati alpini nell'Europa preindustriale (1650-1850), Torino, Rosenberg \& Sellier, 2007.

Habermas, Rebekka, Frauen und Männer des Büergertums. Eine Familiengschichte (1750-1850), Göttingen, Vandenhoeck \& Ruprecht, 2000.

Hartog, François, Revel, Jacques (dir.) Les Usages politiques du passé, Paris, EHESS, 2001.

Hradil, Stefan, Soziale Ungleichheit in Deutschland (8. Auflage), Wiesbaden, VS Verlag für Sozialwissenschaften, 2005 (2001).

Imízcoz, José Maria, «Las redes sociales de las élites. Conceptos, fuentes y aplicaciones", in Las élites en la época moderna: la Monarquía española, éd. par Enrique Soria Mesa, Juan Jesùs Bravo Caro, José Miguel Delgado Barrado, Córdoba, Universidad de Córdoba, 2009, t. 1, p. 77-111.

Lemercier, Claire, "Analyse de réseaux et histoire», Revue d'histoire moderne et contemporaine, 52 (2005/2), p. 88-112. 
Levi, Giovanni, L'eredità immateriale. Carriera di un esorcista nel Piemonte del Seicento, Torino, Einaudi, 1985.

—, "Tre storie di famiglia: i fronti parentali», in L'eredità immateriale. Carriera di un esorcista nel Piemonte del Seicento, Giovanni Levi, Torino, Einaudi, 1985, p. 44-82.

—, "I pericoli del geertzismo", Quaderni storici, 58 (1985), p. 269-277.

—, "Les usages de la biographie», Annales ESC, 6 (1989), p. 1325-1336.

-, "Family and Kin - a few thoughts", Journal of Family History, 15 (1990/4), p. 567-578.

—, "On Microhistory", in New Perspectives on Historical Writing, ed. by Peter Burke, Cambridge/Oxford, Polity Press, 1991, p. 93-113.

—, «Il passato remoto. Sull'uso politico della storia», Nuvole, 10 (2000), p. 16-22.

Lipp, Carola, «Kinship Networks, Local Government, and Elections in a Town in Southwest Germany, 1800-1850 », Journal of Family History, 30 (2005/4), p. 347-365.

Loriga, Sabina, Le petit $X$ : de la biographie à l'histoire, Paris, Seuil, 2010.

Mandrou, Robert, Introduction à la France moderne, 1500-1640. Essai de psychologie historique, Paris, Albin Michel, 1961.

Medick, Hans, "Missionare im Ruderboot?" Ethnologische Erkenntnisweisen als Herausforderung an die Sozialgeschichte», Geschichte und Gesellschaft, 10 (1984), p. 295-319.

Ménétra, Jacques-Louis, Journal de ma vie. Jacques-Louis Ménétra, compagnon vitrier au XVIIIe siècle, éd. par Daniel Roche, Paris, Montalba, 1982.

Merzario, Raul, Il paese stretto. Strategie matrimoniali nella diocesi di Como. Secoli XVI-XVIII, Torino, Einaudi, 1981.

Morgenstern, Oskar, Neumann, John von, Theory of Games and Economic Behavior, Princeton, Princeton University Press, 1953.

Muir, Edward, Ruggiero, Guido, Microhistory and the Lost Peoples of Europe: Selections from Quaderni Storici, Baltimore, Johns Hopkins University Press, 1991.

Padgett, John F., Ansell, Christopher K., "Robust Action and the Rise of the Medici, 1400-1434", American Journal of Sociology, 98 (1993/6), p. 1259-1319.

Raggio, Osvaldo, Faide e parentela. Lo stato genovese visto dalla Fontanabuona, Torino, Einaudi 1990. 
Ramella, Franco, Terra e telai. Sistemi di parentela e manifattura nel Biellese dell'Ottocento, Torino, Einaudi, 1984.

Redond, Pietro, Galileo eretico, Torino, Einaudi, 1983.

Restif de la Bretonne, Nicolas Edme, Monsieur Nicolas ou le coeur humain dévoilé: mémoires intimes, Paris, I. Liseux, 1883.

Revel, Jacques, "Microstoria», in Historiographies I. Concepts et débats, éd. par Christian Delacroix, François Dosse, Patrick Garcia, Nicolas Offenstadt, Paris, Gallimard, 2010, p. 529-534, (Folio histoire).

Revel, Jacques (éd.), Jeux d'échelles. La micro-analyse à l'expérience, Paris, Gallimard-Seuil, 1996.

Rosé, Isabelle, «Reconstitution, représentation graphique et analyse de réseaux de pouvoir au haut Moyen Âge. Approche des pratiques sociales de l'aristocratie à partir de l'exemple d'Odon de Cluny (†942)", Redes, 21 (2011/5), p. 199-272.

Sabean, David Warren, Power in the Blood. Popular Culture and Village Discourse in Early Modern Germany, Cambridge, Cambridge University Press, 1984.

-, Kinship in Neckarhausen, 1700-1870, Cambridge, Cambridge University Press, 1998.

—, "Kinship and Class Dynamics in Nineteenth-Century Europe», in Kinship in Europe: Approaches to the Long-Term Development (1300-1900), ed. by David Warren Sabean, Simon Teuscher, Jon Mathieu, New York/Oxford, Berghahn Books, 2007, p. 301-313.

Sabean, David Warren, Teuscher, Simon, «Introduction», in Kinship in Europe: Approaches to the Long-Term Development (1300-1900), ed. by David Warren Sabean, Simon Teuscher, Jon Mathieu, New York/Oxford, Berghahn Books, 2007, p. 1-32.

Saussure, Albertine de, "Chronique de sa vie quotidienne, et récit de la cour que Jacques Necker lui fait et à laquelle son père lui a ordonné de résister", Le Mois suisse, (1939-1940)(rédigé en 1783).

Schnyder-Burghartz, Albert, Alltag und Lebensformen auf der Basler Landschaft um 1700. Vorindustrielle, ländliche Kultur und Gesellschaft aus mikrohistorischer Perspektive. Bretzwil und das obere Waldenburger Amt von 1690 bis 1750, Basel, Liestal, 1992.

Stuber, Martin, Hächler, Stefan, Lienhard, Luc (Hrsg.), Hallers Netz. Ein Europäischer Gelehrtenbriefwechsel zur Zeit der Aufklärung, Basel, Schwabe, 2005. 
Thompson, Edward Palmer, The Making of the English Working Class, London, Victor Gollancz, 1963.

-, Società patrizia, cultura plebea: otto saggi di antropologia storica sull'Inghilterra del Settecento, trad. par Sabina Loriga et al., Torino, Einaudi, 1981.

Torre, Angelo, Luoghi. La produzione di località in età moderna e contemporanea, Roma, Donzelli, 2011.

Trepp, Anne-Charlotte, Sanfte Männlichkeit und selbständige Weiblichkeit, Frauen und Männer im Hamburger Bürgertum zwischen 1770 und 1840, Göttingen, Vandenhoeck \& Ruprecht, 1996.

Trivellato, Francesca, «Is there a Future for Italian Microhistory in the Age of global History?", Californian Italian Studies, 2 (2011), p. 1-24, en ligne: http://escholarship.org/uc/item/0z94n9hq.

ZAUGG, Roberto, "Johann Peter Oettinger entre histoire atlantique, mémoire familiale et littérature coloniale (XVIIe-XXe siècles). Un projet de recherche», Revue de l'Institut français d'histoire en Allemagne, 6 (2014).

Zemon Davis, Natalie, "Les Conteurs de Montaillou», Annales ESC, 34 (1979), p. 61-73. 


\section{ANNEXES \\ EXTRAITS DE TRAVAUX CITÉS}

Ginzburg, Carlo, Le fromage et les vers. L'univers d'un meunier du XVI siècle, trad. par Monique Aymard, Paris, Flammarion, 1980, p. 15-16.

/p. 15/ Quand des équipes entières de chercheurs se lancent dans d'immenses entreprises d'histoire quantitative des idées ou d'histoire religieuse sérielle, proposer une enquête minutieuse sur un meunier peut sembler paradoxal et absurde: presque un retour au métier à main à une époque de métiers automatiques. Il est symptomatique que la possibilité même d'une pareille enquête ait été exclue d'avance par quelqu'un qui, comme F. Furet, a soutenu que la réintégration des classes inférieures dans l'histoire générale ne peut se faire que sous le signe du «nombre et de l'anonymat » à travers la démographie et la sociologie, et "l'étude quantitative des sociétés du passé» ${ }^{1}$. Même si les historiens ne les ignorent plus, les classes inférieures seraient de toute façon condamnées à rester «silencieuses».

Mais si la documentation nous offre la possibilité de reconstituer non seulement des masses indistinctes, mais aussi des personnalités individuelles, il serait absurde de l'écarter. Elargir vers le bas le concept historique "d'individu" n'est pas un mince objectif. Le risque existe, assurément de tomber dans l'anecdote et dans l'histoire événementielle tant décriée, qui n'est pas seulement, ni nécessairement, une histoire politique./p. 16/ Mais le risque n'est pas, pourtant, inévitable. Des études biographiques ont démontré que chez un individu médiocre, en lui-même privé de relief et pour cette raison précisément représentatif, on peut observer comme dans un microcosme les caractéristiques d'une entière couche sociale à une époque historique donnée - par exemple la noblesse autrichienne ou le bas clergé anglais au XVIIe siècle.

Mais est-ce le cas de Menocchio? Pas du tout. Impossible de le considérer comme un Paysan «typique» (dans le sens de «moyen» ou de «statistiquement fréquent») de son temps: son isolement relatif dans le village est clair. Aux yeux de ses concitoyens, Menocchio était un homme au moins en partie différent des autres. Mais cette singularité avait des limites bien précises. On ne sort de la

I. François Furet, «Pour une définition des classes inférieures à l'époque moderne», Annales ESC, 18 (1963), p. 459. 
culture de son temps et de sa classe que pour entrer dans le délire de la noncommunication. Comme la langue, la culture offre à l'individu un horizon de possibilités latentes - une cage flexible et invisible dans laquelle exercer sa propre liberté conditionnelle. Avec clarté et une lucidité rare, Menocchio a articulé le langage historiquement à sa disposition. C'est pourquoi on peut retrouver dans ses confessions, sous une forme particulièrement, nette et presque exaspérée, une série d'éléments convergents qui, dans une documentation analogue, contemporaine ou de peu postérieure, apparaissent dispersés ou à peine mentionnés. Quelques sondages confirment l'existence d'éléments que l'on peut ramener à une culture paysanne commune. En conclusion, même un cas limite - et Menocchio en est certainement un - peut se révéler représentatif. Soit négativement - car il aide à préciser ce qu'il faut entendre, dans une situation donnée, par "statistiquement fréquent». Soit positivement - car il permet de circonscrire les possibilités latentes de quelque chose (la culture populaire) qui ne nous est connu qu'à travers une documentation fragmentaire et déformée, provenant presque intégralement des «archives de la répression ».

Levi, Giovanni, Le pouvoir au village. Histoire d'un exorciste dans le Piémont du XVII siècle, trad. par Monique Aymard, Paris, Gallimard, 1989 (1985), p. 12-13.

/p. 12/ J'ai donc tenté d'étudier un fragment minuscule du Piémont du XVII siècle, en utilisant une technique intensive de reconstruction des événements biographiques de tous les habitants du village de Santena qui ont laissé une trace documentaire. À la longue, toutes les stratégies personnelles et familiales tendent, peut-être, à apparaître émoussées pour se fondre dans un résultat commun d'équilibre relatif. Mais la participation de chacun à l'histoire générale, à la formation et la modification des structures portantes de la réalité sociale, ne peut être évaluée seulement sur la base des résultats perceptibles: au cours de la vie de chacun, d'une manière cyclique, naissent des problèmes, des incertitudes, des choix, une politique de la vie quotidienne qui a son centre dans l'utilisation stratégique des règles sociales.

L'apparition de conflits et de contradictions s'accompagne de la formation continuelle de nouveaux niveaux d'équilibre, assez instables pour être sujets à de nouvelles ruptures. Nous regardons en général une telle société de loin: nous sommes ainsi attentifs à des résultats finaux qui dépassent souvent la possibilité de contrôle des personnes, et leur vie même. Il nous semble que les lois de l'Etat moderne se sont imposées contre des résistances impuissantes et historiquement, à la longue, négligeables. Mais il n’en a pas été ainsi: dans les interstices des systèmes normatifs établis ou en formation, groupes et personnes 
développent leur propre stratégie significative. Celle-ci est capable de marquer la réalité politique d'une empreinte durable, non /p. 13/ d'empêcher les formes de domination, mais de les conditionner et de les modifier.

L'hypothèse dont je suis parti est donc la prise en compte d'une rationalité spécifique du monde paysan, mais non dans les termes génériques d'une réalité culturelle qui serait inconsciente de la société complexe destinée à l'étouffer progressivement. Cette rationalité peut être décrite avec plus de précision si l'on admet qu'elle ne s'exprimait pas seulement dans la résistance à la société nouvelle qui se développait, mais qu'elle visait à réaliser activement une transformation et une utilisation du monde social et naturel: c'est dans ce sens que j'ai utilisé le mot de stratégie.

C'est une rationalité sélective: l'interprétation du système de décision d'un groupe, ou de simples individus, aujourd'hui comme dans le passé s'est trop souvent appuyée sur un schéma fonctionnaliste et néo-classique. Maximisation des résultats fixés à l'avance et minimisation des coûts, disponibilité totale à l'effort tendu vers un objectif. absence d'inertie, insignifiance dans la détermination des buts de l'interaction entre les personnes et du contexte spécifique, cohérence d'intérêts et de mécanismes psychologiques de tous les groupes sociaux, totale disponibilité des informations: ces simplifications de la réalité ne peuvent pas ne pas rendre mécaniques les relations entre individus et normes, entre décision et action. Dans l'histoire racontée ici, les catégories interprétatives sont autres: l'ambiguïté des règles, la nécessité de prendre consciemment des décisions dans des conditions d'incertitude, la quantité limitée d'informations qui permet toutefois d'agir, la tendance psychologique à simplifier les mécanismes de causalité que l'on juge importants pour la détermination des comportements et, enfin, l'utilisation consciente des incohérences entre systèmes de règles et de sanctions.

Revel, Jacques, "L'histoire au ras du sol", in Histoire d'un exorciste dans le Piémont du XVII siècle, Giovanni Levi, Paris, Gallimard, 1989 (1985), p. XXX-XXXII.

/p. XXX/ Admettons qu'en limitant le champ d'observation on fasse surgir des données non seulement plus nombreuses, plus fines, mais qui en outre s'organisent selon des configurations inédites et font apparaître une autre cartographie du social. Quelle peut bien être la représentativité d'un échantillon ainsi circonscrit? Que peut-il nous apprendre qui soit généralisable?

La question a très tôt été posée et elle a reçu des réponses qui n'emportaient guère l'adhésion. Dans un article déjà ancien, Edoardo Grendi avait prévenu l'objection en forgeant un élégant oxymoron: il proposait la notion 
d'«exceptionnel normal ${ }^{2}$. /p. XXXI/ Ce diamant obscur a fait couler beaucoup d'encre. Il exerce la fascination des concepts que l'on souhaiterait pouvoir utiliser si seulement on savait les définir exactement. Faut-il voir dans l'«exceptionnel normal» un écho, tout à fait consonant avec la sensibilité des années d'après 1968, de la conviction que les marges d'une société en disent plus sur celle-ci que son centre? Que les fous, les marginaux, les malades, les femmes (et l'ensemble des groupes dominés) sont les détenteurs privilégiés d'une sorte de vérité sociale? Faut-il le comprendre en un sens assez différent, celui d'un écart significatif (mais à quoi)? Ou encore comme une première formulation du paradigme de l'indice plus tard reproposé par Carlo Ginzburg? Ces diverses hypothèses, et d'autres encore, sont peut-être vraies; elles sont en tout cas plausibles; et il se peut même qu'elles aient coexisté dans la pensée de Grendi, sous réserve qu'elles soient compatibles entre elles (ce qui n'est pas assuré). N'étant point l'auteur de ce mystère épistémologique, je ne feindrai pas de l'organiser et je me garderai de choisir entre ces différentes interprétations. Je note simplement que l'une ou l'autre laisse ouvert le problème de savoir quels enseignements généraux l'on peut espérer tirer d'une enquête locale, ponctuelle, ancrée dans son exceptionnelle même.

Dans ce débat ouvert, le livre de Giovanni Levi me paraît apporter un certain nombre de réponses qui déplacent utilement l'argumentation. Il rappelle en premier lieu qu'on peut penser l'exemplarité d'un fait social autrement qu'en termes rigoureusement statistiques. Le deuxième chapitre, consacré aux stratégies développées par trois familles de métayers de Santena, opère un choix parmi quelques centaines d'autres cas possibles, qui ne font l'objet d'aucun traitement comparable, mais qui sont tous présents dans le fichier prosopographique. La démarche n’a donc pas consisté à référer ces trois exemples à la totalité de l'information constituée, mais à en abstraire les éléments d'un modèle. Ces trois biographies familiales, assez fortement contrastées, suffisent à faire apparaître/p. XXXII/ des régularités dans les comportements collectifs d'un groupe social particulier. Tester la validité du modèle consistera donc non pas en une vérification du type statistique, mais dans sa mise à l'épreuve dans des conditions extrêmes, lorsqu'une ou plusieurs des variables qu'il inclut sont soumises à des déformations exceptionnelles. La constitution d'un fichier systématique est précisément ce qui rend possible une vérification de ce type.

Le parcours sinueux, compliqué, proposé par Levi me paraît en outre avoir le mérite de ne jamais refermer le champ restreint de l'enquête lourde sur luimême, mais de jouer de façon raisonnée sur ce que l'on pourrait nommer des

2. Edoardo Grendi, «Microanalisi e storia sociale», Quaderni Storici, 33 (1972), p. 506-520. 
variations d'échelles d'observation ${ }^{3}$. De Santena même, nous apprenons en gros tout ce qu'on peut savoir aujourd'hui sur une période d'un demi-siècle: tout, c'est-à-dire beaucoup sur les réalités économiques et sociales, et quelque chose, souvent laissé à l'état de traces plus fugitives, sur la dynamique politique qui travaille cette communauté. Mais l'intelligence de cette aventure paroissiale n'est pas saisissable au seul niveau local. C'est en recourant de façon systématique à des variations de la focale, qui permettent d'inscrire Santena dans une série de contextes emboîtés, que cette histoire prend sens peu à peu.

Levi, Giovanni, «Les usages de la biographie», Annales ESC, 6 (1989), p. $1333-1334$.

/p. 1333/ C'est avant tout un problème d'échelle et de point de vue: si l'accent porte sur la destinée d'un personnage - et non sur l'ensemble d'une situation sociale - afin d'interpréter le réseau de relations et d'obligations extérieures dans lequel il s'insère, il est tout à fait possible de concevoir différemment la question du fonctionnement effectif des normes sociales. De manière générale, les historiens tiennent pour acquis que tout système normatif subit des transformations dans le temps, mais qu'à un moment donné il devient pleinement cohérent, transparent et stable. Il me semble, au contraire, que l'on devrait s'intéresser davantage sur l'ampleur réelle de la liberté de choix. Bien entendu, cette liberté n'est pas absolue: culturellement et socialement déterminée, limitée, patiemment conquise, elle demeure toutefois une liberté consciente que les interstices inhérents aux systèmes généraux de normes laissent aux acteurs. Aucun système normatif n'est, de fait, assez structuré pour éliminer toute possibilité de choix conscient, de manipulation ou d'interprétation des règles, de négociation. Il me semble que la biographie constitue, à ce titre, le lieu idéal pour vérifier le caractère interstitiel - et néanmoins important - de la liberté dont disposent les agents, comme pour observer la façon dont fonctionnent /p. 1334/ concrètement des systèmes normatifs qui ne sont jamais exempts de contradictions. On retient ainsi une perspective différente - mais non contradictoire - de ceux qui choisissent de souligner davantage les éléments de détermination, nécessaires et inconscients, comme le fait, par exemple, Pierre Bourdieu. Il y a relation permanente et réciproque entre biographie et contexte; le changement est précisément la somme infinie de ces interrelations. L'intérêt de la biographie est de permettre une description des normes et de leur fonctionnement effectif,

3. Ces lignes doivent beaucoup aux discussions que j'ai eues sur ce thème avec Bernard Lepetit, autour de questions qui nous sont apparues communes. Je suis seul responsable, cela va de soi, de la lecture avancée ici. 
celui-ci n'étant plus présenté seulement comme le résultat d'un désaccord entre règles et pratiques, mais tout autant comme celui des incohérences qui autorisent la multiplication et la diversification des pratiques. Il me semble qu'on évite ainsi d'aborder la réalité historique à partir d'un schéma unique d'actions et de réactions et que l'on montre, au contraire, que l'inégale répartition du pouvoir, aussi grande et aussi coercitive soit-elle, n'est pas sans offrir une certaine marge de manœuvre aux dominés; ces deniers peuvent dès lors imposer aux dominants des changements non négligeables. Il ne s'agit peut-être que d'une nuance, mais il me semble toutefois qu'on ne peut analyser le changement social là où l'on n'a pas préalablement reconnu l'existence irréductible d'une certaine liberté vis-à-vis des formes rigides et des origines de la reproduction des structures de la domination.

Sabean, David W., Power in the Blood. Popular Culture and Village Discourse in Early Modern Germany, Cambridge, Cambridge University Press, 1984, p. 28-30

/p. 28/ There are two guiding principles in my notion of 'community', and here I mean the small town or village community - the Gemeinde. Community is a matter of mediations and reciprocities and it cannot be analysed apart from Herrschaft. What makes community possible is the fact that it involves a series of mediated relationships. One central form of mediation, of course, is provided by property - the access to resources, the apportionment of rights and claims, and the acceptance of obligations and duties. Other forms of mediation can be found within the spheres of production and exchange or in the sphere of social value - the way, for example, honor is allocated within the constant reflection of villages upon each other. By emphasizing relationships, it can be seen that community includes both negative and positive elements, both sharing and conflict. From the theologian's or psychologist's point of view, then, community exists where not just love but also frustration and anger exist ${ }^{4}$. In several chapters, we will find that villagers grasped community most centrally under the terms 'envy' and 'hate'.

In some respects there are many communities as there are mediated relations. This fact is recognized, for example, in such terms as 'marital community'. It suggests the use of the concept for those relationships which contain multiple links and are structured over time. The issues in most of the examples

4. This is the implication, for example, in the article 'Person' in Die Religion in Geschichte und Gegenwart, 3rd edn, vol. 5 (Tübingen, 1961), p. 234. The discussion of 'person' as constituted within mediated relationships is also valid for 'community'. 
in this book is to what degree a community /p. 29/ is a community; in what way a collectivity such as a village or a neighbourhood is bound together through mediated relationships involving aid, conflict, aggression, and sharing. In the way that we confront the reality of village life, we see that community was not something 'pre-modern', unchanging, structural, but was constructed, changed with time, and can onlv be grasped as historical process because those elements through which relations were constructed, whether 'real' or symbolic resources, were constantly in movement.

Notions such as Gemeinschaft and Gesellschaft when ordered temporally often cloud important issues in so far as history can only enter when a break in forms of society takes place. It is common with such dichotomous concepts to introduce process only in the transformation from one structure to the other ${ }^{5}$. It is forgotten to begin with that reproduction is also a process and as much subject to historical effort as any other process. But more than that, concepts of this kind have to be useful for gasping both real changes and, where relevant, forces for structural stasis. Since we know that villages were constantly altering their structural relations as the nature of state institutions changed, then concepts are necessary for dealing with this matter. But even here Herrschaft appears to be dynamic while 'community' tends to be historically flat. Perhaps we can avoid historical flatness by stressing changes in the way relations between people were mediated. If, for example, a peasant can get along with one horse for plowing, then relations between households are in part determined by access to this important instrument of production. Horseless peasants will be caught up in daily relationships of dependence in order to ensure adequate plowing at the right time. But if, as was common in Württemberg, many villagers in the late eighteenth century shifted over to cattle, relations would have undergone structural alterations. Two cows or oxen were necessary for plowing, and practically no one could make up a plow team on their own. As a result, interdependence in production had to take quite different forms.

What is common in community is not shared values or common understanding so much as the fact that members of a community are engaged in the same argument, the same raisonnement, the same Rede, the same discourse, in which alternative strategies, misunderstandings, conflicting goals and values are threshed out. In so far as the individuals in a community may all be caught up in different webs of connection to the outside, no one is bounded in his relations by the community, and boundedness is not helpful in describing what

5. The same problem appears in Ivan Illich's recent book, Gender (New York, 1982). On the issue of 'community' as an analytical concept and 'tradition' as something that needs to be exlplained, see useful overview by Sydel Silverman, 'The Peasant Concept in Autobiography', Journal of Peasant Studies, 7 (1979), p. 49-69, especially p. 56 sq., p. 63 sq. 
community is ${ }^{6}$. What /p. 30/ makes community is the discourse. In so far as part or much of what people do does not enter in any way into the discourse, to what extent they simply pass each other by. Yet, the problem of inside and outside is complex. What one does outside of community may have enormous implications for one's position inside, and vice versa.

Medick, Hans, "Missionnare im Ruderboot"? Ethnologische Erkenntnisweisen als Herausforderung an die Sozialgeschichte", Geschichte und Gesellschaft, 10 (1984), p. 313-314.

/p. 313/ Die Vergegenwärtigung der Möglichkeiten und Grenzen der teilnehmenden Beobachtung der Ethnologen und der eigentümlichen interpretativen Kraft kultureller Äußerungs- und Ausdrucksweisen bedeutet jedoch nicht nur eine Herausforderung, unsere Vorstellungen von Inhalten, sondern auch von den Erkenntnisweisen der Sozialgeschichte zu überprüfen. Jedenfalls stellt sich das Verhältnis von Theorie und Geschichte, von Konstruktion des Forschers und Erfahrung der „Sache“, von Subjekt und Objekt im Forschungsprozeß im Lichte dieser Herausforderungen anders dar, als dies in einigen ausgearbeiteten Positionen der „historischen Sozialwissenschaft" der Fall ist. In Analogie zu den Formulierungen von Geertz ließe sich sagen, daß die „eingeborene Theori“" des historischen Subjekte hier ebenso wichtig wird, wie die darauf bezugnehmende Theorie des Historikers.

Das bedeutet zunächst und vor allem eine Infragestellung der Quelle als eines bloßen Dokuments, aus dem Fakten zu entnehmen sind, die sich vor allem dazu eignen, quantitativ in Serien verarbeitet zu werden. Auch eine Quelle wird verstärkt auf ihre interpretative Dimension in Bezug auf die historisch-gesellschaftliche Wirklichkeit zu befragen sein, aus der sie stammt. So ist am Beispiel der Arbeit an den Inventurlisten württembergischer Haushalte des 18. und 19. Jahrhunderts etwa zu sehen, daß die dort minutiös aufgeführten Gebrauchsgegenstände und Arbeitswerkzeuge nicht nur Informationen über „Haushaltsausstattungen“ geben, sondern diese Gegenstände auch als symbolische Zeugnisse materieller Kultur und alltäglicher Repräsentation ernst und Lebensweisen zu befragen sind ${ }^{7}$.

6. See the simulating article by Joan Vincent, 'Agrarian Society as Organized Flow: Process of Development Past and Present', Peasant studies, 6 (1977), p. 56-65.

7. Hierzu sind eigene Arbeiten in Vorbereitung. Vgl. zu einigen Möglichkeiten der auswertung H. Medick, Weben, Überleben u. Widerstand im alten Laichingen, in: Schwäbische Heimat 35.1984 und A. Bischoff-Luithlen, Der Schwabe u. sein Häs, Stuttgart 1982. 
Die „eingeborene Theorie der Historischen Subjekte“ ernstzunehmen, diese Arbeit enthält aber durchaus auch konkrete methodische Ansprüche /p. 314/ und Möglichkeiten. Sie sind z. B. in historischen Mikro-Analyse kleiner gesellschaftlicher Einheiten einzulösen - etwa eines Dorfes, einer Stadt oder eines Betriebes. Hier wird auf der Basis der namentlichen Erhebung der Gesamtbevölkerung aus verschiedenen Quellen deren zusammenfassende Analyse versucht. Ziel ist hierbei, die Untersuchung der alltäglichen Lebens-, Arbeits- und Erfahrungssituation der verschiedenen Schichten und Gruppen in Dorf bzw. Stadt mit der Analyse der sozialen und ökonomischen Lebensverhältnisse zu verbinden, einschließlich der Ursachen ihres Wandels und ihrer Beharrung ${ }^{8}$.

Erst von hier aus dürfen sich auf neue Weise Einblicke in den Zusammenhang umfassenderer gesellschaftlicher Transformationsprozesse eröffnen, z. B. der langfristigen Übergänge zum agrarischen und industriellen Kapitalismus.

Ein solches Verfahren könnte als ein - aber keineswegs als das einzige sozialhistorische Gegenstück zur ethnologischen „thick description“ bezeichnet werden. Wenigstens wird es so möglich, die in den Quellen vorfindlichen, häufig doch recht dünnen Geschichten zu dickeren bzw. dichteren Beschreibungen anzureichern ${ }^{9}$. Die Fragmentierung der einzelnen historischen Quellen wird so wenigstens teilweise überwunden und damit auch eine selektive Perspektive, welche sozialgeschichtliche Quellen als obrigkeitliche Zeugnisse durchgängig kennzeichnet und die, auf die historische Darstellung übertragen, oft zu einer fragmentierten Sichtweise des historischen Zusammenhangs führt. Eine so verstandene mikro-analytische „dichte Beschreibung" bliebe also auf dem Weg der wechselseitigen Ergänzung der selektiven Perspektive der einzelnen Quelle nicht bei sich, sie verharrte nicht im historischen Detail, sondern führte von sich aus über sich hinaus zu den systematischen Problemen.

8. S. hierzu den originellen kleinen Essay: C. Ginzburg, C. Poni, «La microhistoire", Le Debat, 17 (1981), p. 133-136.

9. Manfred Thaller hat hierfür mit seinem Clio-System quellenorientierter Datenverarbei- tung wichtige theoretische und praktische Voraussetzungen geschaffen. Ähnlich den Ein- sichten, die sich aus der „thick description“ der Ethnologen ergeben, geht sein Verfahren von der Mehrdeutigkeit und Unscharfe historischer Quellen aus (die sich gegen die herkömmlichen, a priori auf Eindeutigkeit orientierten, methodischen und konzeptuellen "Zugriffe" der systematischen Sozialwissenschaften sperrt) und versucht, dieser entscheidenden Voraussetzung historischer Arbeit und Interpretation gerecht zu werden: ders., Ungefähre Exaktheit. Theoretische Grundlagen u. praktische Möglichkeiten einer Formulierung historischer Quellen als Produkte „unscharfer" Systeme, in: Sonderheft Conceptus 1984; ders., Automation on Parnassus. Clio - A Databank Oriented System for Historians, in: Historical Social Research/Historische Sozialforschung 15.1980, S. 40-65. 
Grendi, Edoardo, "Ripensare la microstoria?», Quaderni storici, 86 (1994), p. 547-548.

/p. 547/ Le elaborazioni teorico-metodologiche di Carlo Ginzburg - sempre così tese nella loro chiave di suggestivo alternativismo - mi sono sempre parse strettamente connesse col suo specifico lavoro storiografico, che si muove per interno nell'ambito del problema storico delle "forme culturali». E questo è senza dubbio il segno di una forte onestà esegetica: la coerenza con se stesso e l'«autorivelazione». Non mi pare infatti che Ginzburg sia attivamente e analiticamente interessato a trovare le mediazioni con il «sociale», le «relazioni interpersonali ": il discorso rimane interno alle forme espressive, al complesso rapporto fra alta e bassa cultura, all'analisi e alla ricostruzione dei rapporti e nessi fra quelle «forme» e le loro filiazioni. È noto che uno dei più vistosi trend della storiografia attuale è l'interesse per l'espressività-rappresentazione: nel caso esterno la "fonte» diviene "testo" e la realtà storica è una illusione ${ }^{10}$. In ogni caso non ci si difende da questo relativismo trascurando le forme espressive e gli assunti di interpretazione storica che esse pongono. A me pare comunque che questa difesa analitica della realtà storica può essere organizzata meglio integrando lo studio delle forme con l'analisi storico-sociale volta a ricostruire dei processi di cui azioni e espressioni sono componenti fondamentali: un'immagine non è solo figlia di altre immagini, è anche connessa con una situazione che essa esprime e organizza. Lo storico può fruttuosamente immaginare et sperimentare schemi interpretativi che gli consentano di rendere intelligibili quei processi. Tanto più che, a tal fine, lo soccorre la tradizione delle scienze sociali : si tratta di modificare, adattare e anche inventare procedure di analisi, ma non necessariamente di costruire tutto percorso stretto et serrato. Questo confronto non è avvenuto nell'ambito /p. 548/ della microstoria, non tanto per definire un'ortodossia, quanto per creare una dialettica alta. È tutto sommato strano che la doppia anima della microstoria, chiara fin dall'inizio, non abbia dato luogo a un processo di chiarificazione o quanto meno di discussione. Come spesso accade, la rinuncia a un «impegno alto» spiega la fine di un'esperienza collettiva.

Per fortuna tuttavia non si tratta di «ripensare» e rilanciare nulla. Oggi «ripensare la microstoria» significa cercare di fame la storia, ed è insufficiente a tal fine collocarla unicamente nella situazione della storiografiche più vive e analiticamente più ricche, come pratica fondamentalmente che quella opzione rappresenta l'occasione per un arricchimento dei significati dei processi storici attraverso un fondamentale rinnovamento delle categorie interpretative e la loro verifica sperimentale. È ben probabile in questo senso che i microstorici italiani

Io. Si veda il dibattito sul post-modernism sui recenti numeri di «Past and Present». 
abbiano molti fratelli nel mondo che ancora non conoscono. Il passaggio dalla storiografia alla "pratica storiografica a forte consapevolezza teorica» e, su questo terreno, il confronto può continuare: la storia diventa una scienza sociale che si costruisce sulle dimensioni del tempo e dello spazio. 